\title{
Coordinated analyses of hydrated Kuiper Belt dust
}

\author{
LINDSAY P. KELLER ${ }^{1}$ AND CHRISTOPHER J SNEAD ${ }^{2}$
}

${ }^{1}$ NASA Johnson Space Center

${ }^{2}$ Jacobs-JETS

Presenting Author: lindsay.p.keller@nasa.gov

We report on coordinated mineralogical, chemical, and isotopic analyses of hydrated carbon-rich interplanetary dust particles (IDPs). We focus on hydrated IDPs that contain high solar flare track densities consistent with an origin in outer Solar System bodies such as Edgeworth-Kuiper belt objects (EKBOs) [1]. We are searching for evidence of the heavy water reservoir predicted by self-shielding models [2]. The IDPs were embedded in sulfur and microtome thin sections were obtained for Fouriertransform infrared (FTIR), transmission electron microscopy (TEM), and nanoSIMS analyses. The IDPs were only partly microtomed and the remainder was extracted from the sulfur and pressed into In or $\mathrm{Au}$ for high precision $\mathrm{O}$ isotopic analyses.

The hydrated IDPs in this study are compact low-porosity objects with a mineralogy dominated by fine-grained saponite and lesser serpentine, $\mathrm{Fe}-\mathrm{Ni}$ sulfides, $\mathrm{Mg}$-Fe carbonates, rare crystalline silicates, and abundant organic matter. The $\mathrm{C}$ in the IDPs is abundant (typically $>4 \mathrm{X} \mathrm{CI}$ ) and occurs as isolated nanoglobules and finely disseminated within the phyllosilicate matrix of the IDPs. TEM analyses of the nanoglobules show Orich, S-depleted rims with higher carbonyl functionality than their cores. FTIR spectra show strong aliphatic $\mathrm{C}-\mathrm{H}$ stretches consistent with the presence of short-chain aliphatic hydrocarbons in these IDPs. The oxygen isotopic compositions measured in 6 IDPs all plot within error of the slope 1 CCAM line [3]. The $\mathrm{D}^{17} \mathrm{O}$ values range from $-23 \%$ up to $+3.4 \%$ with most analyses clustering above the terrestrial fractionation line. The $\mathrm{O}$ isotopic compositions and the high $\mathrm{C}$ contents of these IDPs are distinct from known hydrated carbonaceous chondrites.

The track-rich hydrated IDPs record parent body aqueous alteration effects through interactions with ${ }^{16} \mathrm{O}$-poor $\mathrm{H}_{2} \mathrm{O}$ on a EKBO parent body. The aqueous alteration activity likely resulted from the heat generated by collisional processes among EKBOs given that these objects are believed to have accreted late after the decay of ${ }^{26} \mathrm{Al}[4]$.

References: [1] Keller, L. P. \& Flynn, G. J. (2019): LPSC 50th, \#2525. [2] Clayton, R. N. (2002): Nature, 415, 860. [3] Keller, L. P. \& Snead, C. J. (2021): LPSC 52nd, \#2389. [4] Nakashima, D. et al. (2015): EPSL 410, 54. 